\title{
Diagnosing Notre-Dame after fire
}

\author{
Aline Magnien, director of the Historical Monuments Research Laboratory in Champs-sur-Marne in France, talks \\ to Nature Materials about their work in materials damage assessment serving for the restoration of Notre-Dame \\ de Paris.
}

Please tell us about your scientific background and your team in Historical Monuments Research Laboratory (LRMH).

My education background is in literature, humanism and art history. I obtained my first degree in literature, what we call in France agrégation de lettres, a university-level teaching credential. I have a PhD in history and art on the topic of eighteenth-century sculptures (with an essay published in 2004 by the Voltaire Foundation at Oxford). After working in the Rodin Museum as a head of collections for nine years, I became the director of LRMH in 2015. It's a tradition that the LRMH is directed by an art historian or a curator, as we believe that what we do in restoration and conservation is guided by cultural and art values, and not simply scientific-oriented.

Although I am not a materials scientist, I am always interested in and have worked a lot on materials that are used in the arts. In fact, materials are the backbone of our work in LRMH. The lab is organized in nine departments, each one dedicated to the research or analysis of a certain material or work: stones, metals, woods, concretes, glasses, textile, microbiology, mural paintings and decorated caves. Almost all my colleagues in these different departments are materials scientists. We conduct case studies on deterioration and conservation of cultural heritage, carry out research programmes, and especially provide scientific assistance for conservation and restoration of historical monuments. Having these two missions - research and service - I believe it is a unique lab in Europe and probably in the world. We have strong links with the French administration of historical monuments, and in fact we are part of its central administration, dedicated to providing technical service and advice to curators, architects and restorers who are in charge of the conservation and restoration works of historical monuments.

What is the role that LRMH plays in the project of Notre-Dame restoration?

Our main task is the damage assessment. For the moment we work mostly on the characterization and assessment of stones, but also of metals, stained glasses, as well as

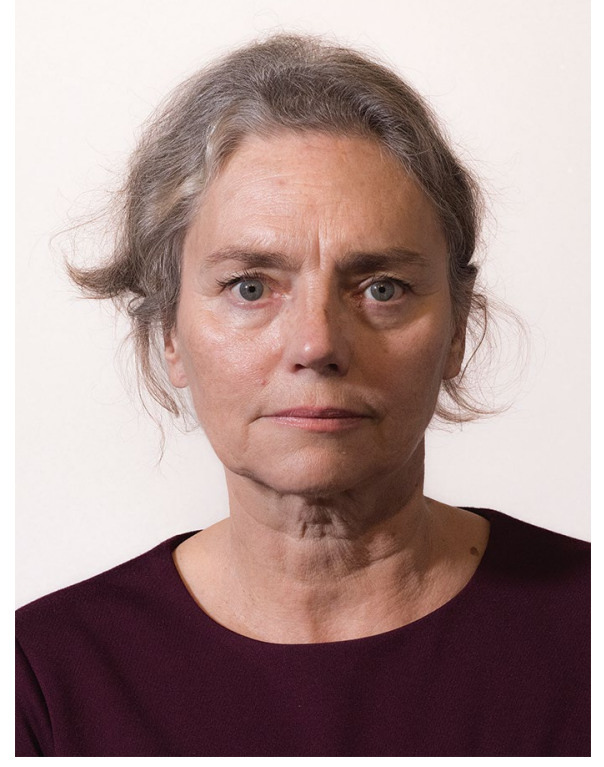

mural paintings, wood and timber. Probably later the department of microbiology would be also involved, since there could be some microbial deterioration once the whole cathedral is closed. During the restoration, we will need to clean the cathedral, the stones, stained glasses and mural paintings.

Having a complete picture of the status of the cathedral after the fire is an essential basis for the restoration. It's impossible to formulate a restoration project without an appropriate evaluation of all the damages. For example, understanding the exact state of the stones is extremely important for the stability and durability of the monument. How long will it take for the stones to dry? How many stones will have to be changed and how? How many pieces can be reused? Also, since the original quarries where the stones were extracted are closed, in order to replace the damaged pieces we have to find new quarries providing stones with exactly the same geological and mechanical qualities. We have to answer all these questions and work with other teams to find solutions.

So the restoration requires substantial collaborative efforts from different teams. How does your team collaborate and coordinate with others?

There are different teams at different levels. We work with the Public
Establishment (EPA), which has been created and responsible for the restoration of Notre-Dame de Paris since the end of 2019. We help the chief architect Philippe Villeneuve and his team on diagnosis, evaluating current status of the cathedral and providing the essential information for them making restoration plans. We also work a lot with archaeologists and curators. All the items after fire like stones, mortars, and metal pieces are being treated as archaeological artefacts. With the archaeologists from the Regional Directorate of Cultural Affairs (DRAC), Île de France, and colleagues from the Centre for Research and Restoration of the Museums of France (C2RMF), we have made a huge selection of items from the vaults of Notre-Dame, which have been saved and analysed, and will be preserved for research opportunities. We help DRAC to take scientific and technical control of the restoration. We are somewhere between architects and curators. We help both of them, for restoration on one hand, and on the other hand for understanding what is proposed for the restoration and whether the actions are good for monuments from scientific perspective.

Many scientists from academic institutions are also interested in the possibilities for their research studies in the aftermath of the fire. All these groups are linked in a structure called Chantier Scientifique Notre-Dame (the Notre-Dame Scientific Project), which associates about 50 research unities and 175 researchers under the direction of both the Ministry of Culture (MC) and the French National Centre for Scientific Research (CNRS). I work with two colleagues from CNRS and one from $\mathrm{MC}$ to coordinate between the academic world and the people who are in charge of the restoration, to establish interdisciplinary cooperation and conduct research activities on the Notre-Dame.

What is the progress of the restoration and what are current challenges?

For the moment, we are still at the stage of damage assessment and presentation of various design plans for the reconstruction. The work has unfortunately been delayed because of many constraints caused, among others, by the discovery of lead 
contamination, weather conditions and the COVID-19 pandemic. The scaffolding, which still stands above the roof of the cathedral, is being removed, since it is dangerous and prevents the architects from closing the sanctuary and making a complete assessment. The steel beams have been installed to support the vault and allow the inspection. During the next six months, we are going to thoroughly characterize all different types of stones, and I expect to get the first results before the end of the year. Other research studies like the ones on woods and metals, which are less critical for the assessment of the structural stability and would not be reused for restoration, are planned for the longer term.

It is not easy, really. But everyone, from the architects to the enterprises working on this field, is doing their best. Very probably, we'll be able to reopen the sanctuary of Notre-Dame as planned in 2024. I am an optimist.

How would you describe this catastrophe from the perspective of research and science?

We try to leverage this tragedy into an opportunity to explore new fields of research and gain a better knowledge of this great monument that we don't really know as well as we believe. For example, we will get more precise information on the different kinds of stones used in Notre-Dame. We will have a clearer idea of the use of metal in the architecture. We will understand better the restoration works made by Viollet-le-Duc, who was a great architect and restored many landmarks in France including Notre-Dame in the middle of the nineteenth century. He probably saved Notre-Dame but actually created a new cathedral controversially. The vault damage has also exposed some areas and details that we could not see before. We can see the 'sectional view' of the vaults and sometimes see all the faces of the stones, which is impossible in a normal time. We can learn more about the way people used different construction materials in the twelfth century. We will probably know better about the timber, sometimes not only for understanding Notre-Dame itself. The way the wood has grown up can provide climate information, for example. This is a chance for investigations on materials science, anthropology, acoustics and other disciplines, which will benefit the whole field of heritage science and beyond.

This is also an occasion for new collaborations. It is exciting to discover new teams at the CNRS or at universities that can offer complementary skills and capabilities. It is probably not easy to work with partners who did not even know each other before, and are from different disciplines with different working styles. We have to learn and figure out how to work together. But it is a challenge well worth it.

\section{What lessons have we learned from the Notre-Dame fire?}

For instance, it became clear that an intervention plan concerted and formed with the fire brigade is really useful. It is one of the reasons why, in a certain way, Notre-Dame has not suffered so much. In fact, the firemen of Paris had made exercises and knew how to respond to the fire in such historical monuments. After 15 April 2019, many monuments and cathedrals in France enforced their own safeguarding plans. A large project has been launched in France by the MC to formulate the security rules (for example, how to take precautions, how to respond quickly to the fire, how to get artistic treasures out in a fire, and what objects to take out first) and to implement new standards for all the cathedrals.

It is maybe too soon to see all the consequences of this catastrophe. But the lesson that is already clear is that we still have plenty of work to understand and preserve these great monuments. We thought we already knew them very well, but we don't.

\section{Interviewed by Xin Li}

Published online: 15 July 2020 https://doi.org/10.1038/s41563-020-0749-x 